\title{
Novel Stability Criterion for Neutral Systems with Mixed Time-Delay
}

\author{
Lianglin Xiong ${ }^{1,2}$, Rong $\mathrm{Li}^{1 *}$, Tao $\mathrm{Wu}^{1}$ and Chen Peng ${ }^{1}$ \\ ${ }^{1}$ School of Mathematics and Statistics, Yunnan University, Kunming, 650091, China \\ ${ }^{2}$ School of Mathematics and Computer Science, Yunnan Minzu University, Kunming, 650500, China \\ ${ }^{*}$ Corresponding author
}

\begin{abstract}
The aim of this paper is to improve stability condition for neutral systems with mixed time-delay. By introducing a novel integral inequality based on the optimization theory, and constructing the novel Lyapunov functionals, an improved delaydependent stability criterion is established. It is shown that the integral inequality obtained in this paper can be utilized to get less conservative delay-dependent stability condition for neutral systems. A numerical example is provided to demonstrate the theoretical result.
\end{abstract}

Keywords-new integral inequality; neutral systems with mixed time-delays; delay-dependent stability

\section{INTRODUCTION}

It is well known that neutral systems are frequently encountered in various engineering systems, including population, ecology, distributed networks containing lossless transmission lines, heat exchangers, and repetitive control. In the past two decades, a great deal of attention have been drawn to the delay-dependent stability problem of neutral dynamical systems (see, e.g., [1]-[31]). Various important methods have been introduced, such as model transformation approach[4], delay partitioning technique[11], discretized Lyapunov functional method[12], free-weighting matrix approach[5], and descriptor system approach[17]. Among those techniques, the Jensen's like inequality has played an important role to obtain delay-dependent stability conditions [21].

However, Jensen's like inequality inevitably introduces an undesirable conservatism, and improving it is always an open problem [25]. Fortunately, it has been improved in [24]-[26] which dealt with single integral terms. Recently, a new multiple integral inequality was introduced following a similar line as in proof of Jensen inequality in [20], and a novel delaydependent stability criterion was established, which has been unfortunately observed that the computational burden is slightly heavy. Actually, [19] observed that the upper bounds of double integral terms should also be estimated if triple integral terms are introduced in the Lyapunov-Krasovskii functional to obtain less conservative conditions. Based on the Wirtinger inequality, [23] improved the double Jensen's like double integral, and a double integral form of the Wirtingerbased integral was introduced. However, they didn't consider the derivative of the integrand directly. We may get another double integral inequality if we consider this case of derivative. Therefore, if one can introduce a less conservative double integral Jensen's like inequality which contains its derivative, the delay-dependent stability results would be improved greatly, which may reduce the complexity of calculation.

In this paper, we will introduce a new integral inequality based on the optimization theory and construction techniques, and then investigate the conventional neutral systems to illustrate the advantages of applying this inequality to obtain delay-dependent stability criterion. The rest of the paper is organized as follows. In Section II, we formulate the problem and the novel inequality is introduced, which is proved to be less conservative than the double integral Jensen's like inequality. In Section III, as an application of the introduced inequality, the stability condition for neutral systems with mixed delay is presented. The sufficient condition is formed in terms of LMIs, which can be easily calculated by Matlab control toolbox [32]. Many cases are compared in the tables based on the conventional numerical example, which is used to show the validity and less conservativeness of our approach in Section IV. The paper is concluded by Section V. The acknowledge is presented in Section VI

\section{PROBLEM STATEMENT AND PRELIMINARIES}

In this paper, we will improve the stability condition for the following neutral system with mixed time-delays:

$$
\left\{\begin{array}{l}
x(t)-C x(t-\tau)=A x(t)+B x(t-h) \\
x\left(t_{0}+\theta\right)=\varphi(\theta), \forall \theta \in[-\max (\tau, h), 0]
\end{array}\right.
$$

where $x(t) \in \mathrm{i}^{n}$ is the state vector, $\tau, h$ are timedelays, $\varphi(\theta)$ is the initial condition function. $A, B, C$ are known matrices. In this paper, we always assume that the spectral radius of $C$ less than 1 .

As is well known, inequalities and Lyapunov functionals are both important to improve the delay-dependent stability condition for neutral systems with mixed time-delays. Accordingly, we will first introduce a novle double integral inequality to deal with the $\int_{-h}^{0} \int_{t+\theta}^{t} w^{\mathrm{T}}(s) R w(s) d s d \theta$ for the derivative of constructed Lyapunov functionals.

Lemma2.1 For a give matrix $R>0$, the following inequality holds for all continuously differentiable function $w \in[t-h, t] \rightarrow \mathrm{i}^{n}:$ 


$$
\int_{-h}^{0} \int_{t+\theta}^{t} w^{\mathrm{T}}(s) R w(s) d s d \theta \geq 6 \Omega^{\mathrm{T}} \mathfrak{R} \Omega
$$

where

$$
\begin{gathered}
R=\left(\begin{array}{ccc}
R & R & -4 R \\
* & 3 R & -8 R \\
* & * & 24 R
\end{array}\right), \\
\Omega^{\mathrm{T}}=\left(w^{\mathrm{T}}(t), \frac{1}{h} \int_{t-h}^{t} w^{\mathrm{T}}(s) d s, \frac{1}{h^{2}} \int_{-h}^{0} \int_{t+\theta}^{t} w^{\mathrm{T}}(s) d s d \theta\right) .
\end{gathered}
$$

Proof. For any continuous function $w(t) \in[t-h, t]$ and which admits a continuous derivative, define the function $z$ by

$$
z(s)=w(s)-\frac{2}{h^{2}} A+\frac{4 g(s)}{h^{3}} B, \text { for } s \in[t-h, t],
$$

where

$$
\begin{gathered}
A=h w(t)-\int_{t-h}^{t} w(s) d s, \quad g(s)=3 t-h-3 s, \\
B=2 \int_{t-h}^{t} w(s) d s+h w(t)-\frac{6}{h} \int_{-h}^{0} \int_{t+\theta}^{t} w(s) d s d \theta .
\end{gathered}
$$

The computation of $\int_{-h}^{0} \int_{t+\theta}^{t} z^{\mathrm{T}}(s) R z(s) d s d \theta$ leads to

$$
\begin{gathered}
\int_{-h}^{0} \int_{t+\theta}^{t} z^{\mathrm{T}}(s) R z(s) d s d \theta= \\
\int_{-h}^{0} \int_{t+\theta}^{t} w^{\mathrm{T}}(s) R w(s) d s d \theta-\frac{4}{h^{2}} \int_{-h}^{0} \int_{t+\theta}^{t} w^{\mathrm{T}}(s) d s d \theta R A \\
+\frac{4}{h^{4}} \int_{-h}^{0} \int_{t+\theta}^{t} d s d \theta A^{\mathrm{T}} R A-\frac{16}{h^{5}} \int_{-h t+\theta}^{0} \int_{t}^{t} g(s) d s d \theta A^{\mathrm{T}} R B \\
+\frac{16}{h^{6}} \int_{-h}^{0} \int_{t+\theta}^{t} g^{2}(s) d s d \theta B^{\mathrm{T}} R B \\
+\frac{8}{h^{3}} \int_{-h}^{0} \int_{t+\theta}^{t} g(s) w^{\mathrm{T}}(s) d s d \theta R B
\end{gathered}
$$

Simple calculation ensure that

$$
\begin{gathered}
\int_{-h}^{0} \int_{t+\theta}^{t} w(s) d s d \theta=A, \quad \int_{-h}^{0} \int_{t+\theta}^{t} d s d \theta=\frac{1}{2} h^{2}, \\
\int_{-h}^{0} \int_{t+\theta}^{t} g(s) d s d \theta=0, \quad \int_{-h}^{0} \int_{t+\theta}^{t} g^{2}(s) d s d \theta=\frac{1}{4} h^{4},
\end{gathered}
$$

and integration by parts ensures that

$$
\int_{-h}^{0} \int_{t+\theta}^{t} g(s) w(s) d s d \theta=-h B .
$$

It thus follows from $R>0$ that

$$
\begin{gathered}
\int_{-h}^{0} \int_{t+\theta}^{t} z^{\mathrm{T}}(s) R z(s) d s d \theta=\int_{-h}^{0} \int_{t+\theta}^{t} w^{\mathrm{T}}(s) R w(s) d s d \theta \\
-\frac{2}{h^{2}} A^{\mathrm{T}} R A-\frac{4}{h^{2}} B^{\mathrm{T}} R B \geq 0
\end{gathered}
$$

More specifically, one can see

$$
\int_{-h}^{0} \int_{t+\theta}^{t} w^{\mathrm{T}}(s) R w(s) d s d \theta \geq \frac{2}{h^{2}}\left(A^{\mathrm{T}} R A+2 B^{\mathrm{T}} R B\right)
$$

or equivalently

$$
\int_{-h}^{0} \int_{t+\theta}^{t} w^{\mathrm{T}}(s) R w(s) d s d \theta \geq 6 \Omega^{\mathrm{T}} \mathfrak{R} \Omega
$$

with $\Re$ and $\Omega$ are defined in Lemma 2.1. This completes the proof.

Remark 2.1 It is worth mentioning that the choice of function $z(s)$ is essential in the proof of Lemma 2.1. On the one hand, the function $g(s)$ should be constructed to mark $\int_{-h}^{0} \int_{t+\theta}^{t} g(s) d s d \theta \times A^{\mathrm{T}} R B=0$, and lead to the right-hand of (5) in a standard quadratic form. Therefore, according to the computation for the integrations, we choose $g(s)=3 t-h-3 s$. On the other hand, to unify the coefficient $\frac{1}{h^{2}}$ of the expression (5), $z(s)$ should contain the coefficient $\frac{1}{h^{3}}$.

Remark 2.2 The inequality in (2) not only contains the information of $w^{\mathrm{T}}(t)$ and $\int_{t-h}^{t} w^{\mathrm{T}}(s) d s$, but also includes the information of $\int_{-h}^{0} \int_{t+\theta}^{t} w^{\mathrm{T}}(s) d s d \theta$. Clearly, inequality (2) of Lemma 2.1 is less conservative than the conventional Jensen's inequality in [28].

\section{MAIN RESULT}

In this section, by constructing a novel Lyapunov functional, and using many inequalities which contain the above introduced inequality, we will give much better delaydependent stability condition.

Theorem 3.1 The system (1) is asymptotically stable if there exist positive definite matrices $P, W_{1}, W_{2}, Q_{1}, Q_{2}, R_{1}, R_{2}$, any symmetric matrices $Y_{i j}, Z_{i j}(i, j=1,2,3,4), \Re_{2 \times 2}$ with ppropriate dimensions satisfying the following linear matrix inequalities

$$
\begin{gathered}
\Psi_{6 \times 6}>0, \\
\varphi_{8 \times 8}<0,
\end{gathered}
$$

where 


$$
\begin{aligned}
& \Psi_{11}=P+Y_{11}+Z_{11}+\frac{4}{\tau} R_{22}+6 h W_{2}+6 \tau W_{1}+\frac{3 h}{2} R_{2}+\frac{3 \tau}{2} R_{1}, \\
& \Psi_{12}=Y_{12}+Z_{12}+\frac{2}{\tau} R_{22}, \Psi_{13}=\tau Y_{13}-2 R_{12}^{\mathrm{T}}-\frac{6}{\tau} R_{22}+6 \tau W_{1}, \\
& \Psi_{14}=6 h W_{2}+\tau^{2} Z_{13}, \quad \Psi_{15}=6 R_{12}^{\mathrm{T}}-24 \tau W_{1}-3 \tau R_{1}+h Y_{14}, \\
& \Psi_{16}=-24 h W_{2}-3 h R_{2}+h^{2} Z_{14}, \Psi_{22}=Y_{22}+Z_{22}+\frac{4}{\tau} R_{22}, \\
& \Psi_{23}=\tau Y_{23}-4 R_{12}^{\mathrm{T}}-\frac{6}{\tau} R_{22}, \Psi_{24}=\tau^{2} Z_{23}, \Psi_{25}=6 R_{12}^{\mathrm{T}}+h Y_{24} \text {, } \\
& \Psi_{26}=h^{2} Z_{24}, \\
& \Psi_{33}=4 \tau Q_{1}+4 \tau R_{11}+\frac{12}{\tau} R_{22}+6 R_{12}+6 R_{12}^{\mathrm{T}}+18 \tau W_{1}+\tau^{2} Y_{33}, \\
& \Psi_{35}=-6 \tau Q_{1}-2 \tau\left(3 R_{11}+\frac{6}{\tau} R_{12}^{\mathrm{T}}\right)-48 \tau W_{1}+h \tau Y_{34}, \\
& \Psi_{44}=4 h Q_{2}+18 h W_{2}+\tau^{4} Z_{33}, \\
& \Psi_{46}=-6 h Q_{2}-48 h W_{2}+h^{2} \tau^{2} Z_{34}, \\
& \Psi_{55}=12 \tau Q_{1}+12 \tau R_{11}+144 \tau W_{1}+6 \tau R_{1}+h^{2} Y_{44}, \\
& \Psi_{66}=12 h Q_{2}+144 h W_{2}+6 h R_{2}+h^{4} Z_{44}, \\
& \varphi_{11}=Q_{1}+Q_{2}-6 R_{1}+R_{11}-6 R_{2}-9 W_{1}-9 W_{2} \\
& +Y_{13}+Y_{13}^{\mathrm{T}}+Y_{14}+Y_{14}^{\mathrm{T}}+h Z_{14}+h Z_{14}^{\mathrm{T}}+\tau Z_{13} \\
& +\tau Z_{13}^{\mathrm{T}}+P A+A^{\mathrm{T}} P+Y_{11} A+A^{\mathrm{T}} Y_{11}^{\mathrm{T}}+Z_{11} A \\
& +A^{\mathrm{T}} Z_{11}^{\mathrm{T}}+R_{12} A+A^{\mathrm{T}} R_{12}^{\mathrm{T}}+A^{\mathrm{T}} R_{22} A+\frac{h^{2}}{2} A^{\mathrm{T}} R_{2} A \\
& +\frac{\tau^{2}}{2} A^{\mathrm{T}} R_{1} A+h^{2} A^{\mathrm{T}} W_{2} A+\tau^{2} A^{\mathrm{T}} W_{1} A \\
& \varphi_{12}=Y_{23}^{\mathrm{T}}-Y_{13}+3 W_{1}+Y_{24}^{\mathrm{T}}+A^{\mathrm{T}} Y_{12}+A^{\mathrm{T}} Z_{12}+h Z_{24}^{\mathrm{T}}+\tau Z_{23}^{\mathrm{T}} \text {, } \\
& \varphi_{13}=3 W_{2}-Y_{14}+R_{12} B+A^{\mathrm{T}} R_{22} B+P B+Y_{11} B+Z_{11} B \\
& +\frac{h^{2}}{2} A^{\mathrm{T}} R_{2} B+\frac{\tau^{2}}{2} A^{\mathrm{T}} R_{1} B+h^{2} A^{\mathrm{T}} W_{2} B+\tau^{2} A^{\mathrm{T}} W_{1} B, \\
& \varphi_{22}=-Q_{1}-R_{11}-9 W_{1}-Y_{23}-Y_{23}^{\mathrm{T}}, \varphi_{23}=Y_{12}^{\mathrm{T}} B-Y_{24}+Z_{12}^{\mathrm{T}} B, \\
& \varphi_{33}=B^{\mathrm{T}} R_{22} B-9 W_{2}-Q_{2}+\frac{h^{2}}{2} B^{\mathrm{T}} R_{2} B+\frac{\tau^{2}}{2} B^{\mathrm{T}} R_{1} B \\
& +h^{2} B^{\mathrm{T}} W_{2} B+\tau^{2} B^{\mathrm{T}} W_{1} B \\
& \varphi_{14}=Y_{12}+Z_{12}+R_{12} C+A^{\mathrm{T}} R_{22} C+P C+Y_{11} C+Z_{11} C \\
& +\frac{h^{2}}{2} A^{\mathrm{T}} R_{2} C+\frac{\tau^{2}}{2} A^{\mathrm{T}} R_{1} C+h^{2} A^{\mathrm{T}} W_{2} C+\tau^{2} A^{\mathrm{T}} W_{1} C,
\end{aligned}
$$

$\varphi_{24}=Y_{22}-R_{12}+Z_{22}+Y_{12}^{\mathrm{T}} C+Z_{12}^{\mathrm{T}} C$, $\varphi_{34}=B^{\mathrm{T}} R_{22} C+\frac{h^{2}}{2} B^{\mathrm{T}} R_{2} C+\frac{\tau^{2}}{2} B^{\mathrm{T}} R_{1} C+h^{2} B^{\mathrm{T}} W_{2} C+\tau^{2} B^{\mathrm{T}} W_{1} C$, $\varphi_{44}=C^{\mathrm{T}} R_{22} C-R_{22}+\frac{h^{2}}{2} C^{\mathrm{T}} R_{2} C+\frac{\tau^{2}}{2} C^{\mathrm{T}} R_{1} C$ $+h^{2} C^{\mathrm{T}} W_{2} C+\tau^{2} C^{\mathrm{T}} W_{1} C$ $\varphi_{15}=\tau Y_{33}+\tau Y_{34}^{\mathrm{T}}+\tau A^{\mathrm{T}} Y_{13}-24 W_{1}-\tau Z_{13}-6 R_{1}$, $\varphi_{25}=36 W_{1}-\tau Y_{33}-\tau Z_{23}, \varphi_{35}=-\tau\left(Y_{34}^{\mathrm{T}}-B^{\mathrm{T}} Y_{13}\right)$, $\varphi_{45}=\tau\left(Y_{23}+C^{\mathrm{T}} Y_{13}\right), \varphi_{55}=-18 R_{1}-192 W_{1}$, $\varphi_{16}=h\left(Y_{34}+Y_{44}+A^{\mathrm{T}} Y_{14}\right)-24 W_{2}-h Z_{14}-6 R_{2}$, $\varphi_{26}=-h Y_{34}-h Z_{24}, \varphi_{36}=36 W_{2}-h\left(Y_{44}-B^{\mathrm{T}} Y_{14}\right)$, $\varphi_{46}=h\left(Y_{24}+C^{\mathrm{T}} Y_{14}\right), \varphi_{66}=-18 R_{2}-192 W_{2}$, $\varphi_{17}=24 R_{1}+60 W_{1}+\tau^{2}\left(A^{\mathrm{T}} Z_{13}+\tau Z_{33}+h Z_{34}^{\mathrm{T}}\right)$, $\varphi_{27}=-60 W_{1}, \varphi_{37}=\tau^{2} B^{\mathrm{T}} Z_{13}, \varphi_{47}=\tau^{2}\left(Z_{23}+C^{\mathrm{T}} Z_{13}\right)$, $\varphi_{57}=48 R_{1}+360 W_{1}-\tau^{3} Z_{33}$, $\varphi_{67}=-h \tau^{2} Z_{34}^{\mathrm{T}}, \varphi_{77}=-144 R_{1}-720 W_{1}, \varphi_{88}=-144 R_{2}-720 W_{2}$, $\varphi_{18}=24 R_{2}+60 W_{2}+h^{2}\left(A^{\mathrm{T}} Z_{14}+h Z_{44}+\tau Z_{34}\right)$, $\varphi_{38}=h^{2} B^{\mathrm{T}} Z_{14}-60 W_{2}, \varphi_{48}=h^{2}\left(Z_{24}+C^{\mathrm{T}} Z_{14}\right)$, $\varphi_{58}=-\tau h^{2} Z_{34}, \varphi_{68}=48 R_{2}+360 W_{2}-h^{3} Z_{44}$, $\varphi_{88}=-144 R_{2}-720 W_{2}$,

Proof. Construct a Lyapunov functional candidate as

$$
V\left(x_{t}, t\right)=\sum_{i=1}^{5} V_{i}\left(x_{t}, t\right)
$$

$$
\begin{aligned}
& V_{1}=x^{\mathrm{T}}(t) P x(t) \\
& V_{2}=\int_{t-\tau}^{t} x^{\mathrm{T}}(s) Q_{1} x(s) d s+\int_{t-h}^{t} x^{\mathrm{T}}(s) Q_{2} x(s) d s \\
& \quad+\int_{t-\tau}^{t}\left(\begin{array}{c}
x(s) \\
x(s)
\end{array}\right)^{\mathrm{T}} \mathfrak{R}\left(\begin{array}{l}
x(s) \\
x(s)
\end{array}\right) d s \\
& V_{3}=\int_{-\tau}^{0} \int_{t+\theta}^{t} x^{\mathrm{T}}(s)\left(\tau W_{1}\right) x(s) d s d \theta \\
& +\int_{-h}^{0} \int_{t+\theta}^{t} x^{\mathrm{T}}(s)\left(h W_{2}\right) x(s) d s d \theta
\end{aligned}
$$


$V_{4}=\int_{-\tau}^{0} \int_{\theta}^{0} \int_{t+\lambda}^{t} x^{\mathrm{T}}(s) R_{1} x(s) d s d \lambda d \theta$

$+\int_{-h}^{0} \int_{\theta}^{0} \int_{t+\lambda}^{t} x^{\mathrm{T}}(s) R_{2} x(s) d s d \lambda d \theta$

$V_{5}=G_{1}^{\mathrm{T}} Z G_{1}+G_{2}^{\mathrm{T}} Y G_{2}$,

where, $Y$ and $Z$ are 4-order matrices with its elements $Y_{i j}, Z_{i j}(i, j=1,2,3,4)$ expressed in theorem 3.1,

$$
G_{1}=\left(\begin{array}{c}
x(t) \\
x(t-\tau) \\
\int_{-\tau}^{0} \int_{t+\theta}^{t} x(s) d s d \theta \\
\int_{-h}^{0} \int_{t+\theta}^{t} x(s) d s d \theta
\end{array}\right), \quad G_{2}=\left(\begin{array}{c}
x(t) \\
x(t-\tau) \\
\int_{t-\tau}^{t} x(s) d s \\
\int_{t-h}^{t} x(s) d s
\end{array}\right)
$$

For the sake of convenience, we set $e_{i} \in{ }^{8 n \times n}(i=1, \quad, 8)$ are elementary matrices, for example

$$
e_{2}^{\mathrm{T}}=\left[\begin{array}{llllllll}
0 & \mathrm{I} & 0 & 0 & 0 & 0 & 0 & 0
\end{array}\right],
$$

and let

$$
\begin{gathered}
e_{0}=A e_{1}+B e_{3}+C e_{4}, \\
\xi^{\mathrm{T}}=\left(\begin{array}{l}
x^{\mathrm{T}}(t), x^{\mathrm{T}}(t-\tau), x^{\mathrm{T}}(t-h), x^{\mathrm{T}}(t-\tau), \\
\frac{1}{\tau} \int_{t-\tau}^{t} x^{\mathrm{T}}(s) d s, \frac{1}{h} \int_{t-h}^{t} x^{\mathrm{T}}(s) d s, \\
\frac{1}{\tau^{2}} \int_{-\tau}^{0} \int_{t+\theta}^{t} x^{\mathrm{T}}(s) d s d \theta, \frac{1}{h^{2}} \int_{-h}^{0} \int_{t+\theta}^{t} x^{\mathrm{T}}(s) d s d \theta
\end{array}\right),
\end{gathered}
$$

First, with the inequality (8) of Lemma 5.1 in [29] and Lemma 2.1, the Lyapunov functionals $V_{2}$ and $V_{3}$ can be scaled as

$$
\begin{aligned}
& V_{2} \geq \xi^{\mathrm{T}} \times\left\{\tau e_{5}^{\mathrm{T}} Q_{1} e_{5}+3 \tau\left(e_{5}-2 e_{7}\right)^{\mathrm{T}} Q_{1}\left(e_{5}-2 e_{7}\right)\right. \\
& +h e_{6}^{\mathrm{T}} Q_{2} e_{6}+3 h\left(e_{6}-2 e_{8}\right)^{\mathrm{T}} Q_{2}\left(e_{6}-2 e_{8}\right) \\
& +\frac{1}{\tau}\left[\begin{array}{ll}
\tau e_{5}^{\mathrm{T}} & e_{1}^{\mathrm{T}}-e_{2}^{\mathrm{T}}
\end{array}\right] \Re\left[\begin{array}{ll}
\tau e_{5}^{\mathrm{T}} & e_{1}^{\mathrm{T}}-e_{2}^{\mathrm{T}}
\end{array}\right]^{\mathrm{T}} \\
& +\frac{3}{\tau}\left[\tau e_{5}^{\mathrm{T}}-2 \tau e_{7}^{\mathrm{T}} \quad-e_{1}^{\mathrm{T}}-e_{2}^{\mathrm{T}}+2 e_{5}^{\mathrm{T}}\right] \Re \\
& \left.\times\left[\begin{array}{cc}
\tau e_{5}^{\mathrm{T}}-2 \tau e_{7}^{\mathrm{T}} & -e_{1}^{\mathrm{T}}-e_{2}^{\mathrm{T}}+2 e_{5}^{\mathrm{T}}
\end{array}\right]^{\mathrm{T}}\right\} \times \xi \\
& V_{3} \geq \xi^{\mathrm{T}} \times\left\{2 \tau\left(e_{1}-e_{5}\right)^{\mathrm{T}} W_{1}\left(e_{1}-e_{5}\right)\right. \\
& +4 \tau\left(e_{1}+2 e_{5}-6 e_{7}\right)^{\mathrm{T}} W_{1}\left(e_{1}+2 e_{5}-6 e_{7}\right) \\
& +2 h\left(e_{1}-e_{6}\right)^{\mathrm{T}} W_{2}\left(e_{1}-e_{6}\right) \\
& \left.+4 h\left(e_{1}+2 e_{6}-6 e_{8}\right)^{\mathrm{T}} W_{2}\left(e_{1}+2 e_{6}-6 e_{8}\right)\right\} \times \xi
\end{aligned}
$$

Following the same line to prove the Jensen inequality in [30], $V_{4}$ can be bounded as

$$
\begin{gathered}
V_{4} \geq \xi^{\mathrm{T}} \times\left\{\frac{6}{\tau^{3}}\left(\frac{\tau^{2}}{2} e_{1}-\tau^{2} e_{7}\right)^{\mathrm{T}} R_{1}\left(\frac{\tau^{2}}{2} e_{1}-\tau^{2} e_{7}\right)\right. \\
\left.+\frac{6}{h^{3}}\left(\frac{h^{2}}{2} e_{1}-h^{2} e_{8}\right)^{\mathrm{T}} R_{2}\left(\frac{h^{2}}{2} e_{1}-h^{2} e_{8}\right)\right\} \times \xi
\end{gathered}
$$

On the other hand, $V_{1}$ and $V_{5}$ can be presented as

$$
V_{1}=\xi^{\mathrm{T}} \times\left\{e_{1}^{\mathrm{T}} P e_{1}\right\} \times \xi
$$

$$
\begin{aligned}
V_{5}= & \xi^{\mathrm{T}} \times\left\{\left[\begin{array}{llll}
e_{1}^{\mathrm{T}} & e_{2}^{\mathrm{T}} & \tau^{2} e_{7}^{\mathrm{T}} & h^{2} e_{8}^{\mathrm{T}}
\end{array}\right] Z\left[\begin{array}{llll}
e_{1}^{\mathrm{T}} & e_{2}^{\mathrm{T}} & \tau^{2} e_{7}^{\mathrm{T}} & h^{2} e_{8}^{\mathrm{T}}
\end{array}\right]^{\mathrm{T}}\right. \\
& \left.+\left[\begin{array}{llllll}
e_{1}^{\mathrm{T}} & e_{2}^{\mathrm{T}} & \tau e_{5}^{\mathrm{T}} & h e_{6}^{\mathrm{T}}
\end{array}\right] Y\left[\begin{array}{lllll}
e_{1}^{\mathrm{T}} & e_{2}^{\mathrm{T}} & \tau e_{5}^{\mathrm{T}} & h e_{6}^{\mathrm{T}}
\end{array}\right]^{\mathrm{T}}\right\} \times \xi
\end{aligned}
$$

From (10) to (14), we can get that

$$
V \geq \xi_{1}^{\mathrm{T}} \Psi \xi_{1}
$$

where

$$
\xi_{1}^{\mathrm{T}}=\left(\begin{array}{l}
x^{\mathrm{T}}(t), x^{\mathrm{T}}(t-\tau), \frac{1}{\tau} \int_{t-\tau}^{t} x^{\mathrm{T}}(s) d s, \frac{1}{h} \int_{t-h}^{t} x^{\mathrm{T}}(s) d s, \\
\frac{1}{\tau^{2}} \int_{-\tau}^{0} \int_{t+\theta}^{t} x^{\mathrm{T}}(s) d s d \theta, \frac{1}{h^{2}} \int_{-h}^{0} \int_{t+\theta}^{t} x^{\mathrm{T}}(s) d s d \theta
\end{array}\right)
$$

According to the inequality (7), the functional (9) is positive definite. Then, the time derivative of $V(t)$ along the trajectories of equation (1) can be firstly computed as follows:

$$
V\left(x_{t}, t\right)=\sum_{i=1}^{5} V_{i}\left(x_{t}, t\right)
$$

with

$$
\begin{gathered}
V_{1}=2 x^{\mathrm{T}}(t) P[A x(t)+B x(t-h)+C x(t-h)] \\
=2 \xi^{\mathrm{T}} e_{1}^{\mathrm{T}} P e_{0} \xi,
\end{gathered}
$$

$$
\begin{gathered}
V_{2}=x^{\mathrm{T}}\left(Q_{1}+Q_{2}\right) x-x^{\mathrm{T}}(t-\tau) Q_{1} x(t-\tau) \\
-x^{\mathrm{T}}(t-h) Q_{2} x(t-h)+\left(\begin{array}{l}
x(t) \\
x(t)
\end{array}\right)^{\mathrm{T}} \mathfrak{R}\left(\begin{array}{l}
x(t) \\
x(t)
\end{array}\right) \\
-\left(\begin{array}{l}
x(t-\tau) \\
x(t-\tau)
\end{array}\right)^{\mathrm{T}} \mathfrak{R}\left(\begin{array}{l}
x(t-\tau) \\
x(t-\tau)
\end{array}\right)
\end{gathered}
$$




$$
\begin{array}{r}
=\xi^{\mathrm{T}}\left[\left(\begin{array}{l}
e_{1} \\
e_{0}
\end{array}\right)^{\mathrm{T}} \mathfrak{R}\left(\begin{array}{l}
e_{1} \\
e_{0}
\end{array}\right)-\left(\begin{array}{l}
e_{2} \\
e_{4}
\end{array}\right)^{\mathrm{T}} \mathfrak{R}\left(\begin{array}{l}
e_{2} \\
e_{4}
\end{array}\right)\right] \xi \\
+\xi^{\mathrm{T}}\left[e_{1}^{\mathrm{T}}\left(Q_{1}+Q_{2}\right) e_{1}-e_{2}^{\mathrm{T}} Q_{1} e_{2}-e_{3}^{\mathrm{T}} Q_{2} e_{3}\right] \xi \\
V_{3}=\xi^{\mathrm{T}}\left[e_{0}^{\mathrm{T}}\left(\tau^{2} W_{1}+h^{2} W_{2}\right) e_{0}-\tau \int_{t-\tau}^{t} x^{\mathrm{T}}(s) W_{1} x(s) d s\right. \\
\left.-h \int_{t-h}^{t} x^{\mathrm{T}}(s) W_{2} x(s) d s\right] \xi
\end{array}
$$

With the inequality (24) of Lemma 5.1 in [29], one can obtain that

$$
\begin{gathered}
V_{3} \leq \xi^{\mathrm{T}}\left[e_{0}^{\mathrm{T}}\left(\tau^{2} W_{1}+h^{2} W_{2}\right) e_{0}-\left(e_{1}-e_{2}\right)^{\mathrm{T}} W_{1}\left(e_{1}-e_{2}\right)\right. \\
-\left(e_{1}-e_{3}\right)^{\mathrm{T}} W_{2}\left(e_{1}-e_{3}\right)-3\left(e_{1}+e_{2}-2 e_{5}\right)^{\mathrm{T}} W_{1}\left(e_{1}+e_{2}-2 e_{5}\right) \\
-3\left(e_{1}+e_{3}-2 e_{6}\right)^{\mathrm{T}} W_{2}\left(e_{1}+e_{3}-2 e_{6}\right) \\
-5\left(e_{1}-e_{2}+6 e_{5}-12 e_{7}\right)^{\mathrm{T}} \times W_{2} \times\left(e_{1}-e_{2}+6 e_{5}-12 e_{7}\right) \\
\left.-5\left(e_{1}-e_{3}+6 e_{6}-12 e_{8}\right)^{\mathrm{T}} \times W_{2} \times\left(e_{1}-e_{3}+6 e_{6}-12 e_{8}\right)\right] \xi \\
V_{4}=\xi^{\mathrm{T}}\left[\frac{1}{2} e_{0}^{\mathrm{T}}\left(\tau^{2} R_{1}+h^{2} R_{2}\right) e_{0}\right] \xi-\int_{-\tau}^{0} \int_{t+\theta}^{t} x^{\mathrm{T}}(s) R_{1} x(s) d s d \theta \\
-\int_{-h}^{0} \int_{t+\theta}^{t} x^{\mathrm{T}}(s) R_{2} x(s) d s d \theta
\end{gathered}
$$

By using the Lemma 2.1, one can see that

$$
\begin{gathered}
V_{4} \leq \xi^{\mathrm{T}}\left[\frac{1}{2} e_{0}^{\mathrm{T}}\left(\tau^{2} R_{1}+h^{2} R_{2}\right) e_{0}\right. \\
-\left(\begin{array}{llll}
e_{1}^{\mathrm{T}} & e_{5}^{\mathrm{T}} & e_{7}^{\mathrm{T}}
\end{array}\right) K_{1}\left(\begin{array}{lll}
e_{1}^{\mathrm{T}} & e_{5}^{\mathrm{T}} & e_{7}^{\mathrm{T}}
\end{array}\right)^{\mathrm{T}} \\
\left.-\left(\begin{array}{llll}
e_{1}^{\mathrm{T}} & e_{6}^{\mathrm{T}} & e_{8}^{\mathrm{T}}
\end{array}\right) K_{2}\left(\begin{array}{llll}
e_{1}^{\mathrm{T}} & e_{6}^{\mathrm{T}} & e_{8}^{\mathrm{T}}
\end{array}\right)^{\mathrm{T}}\right] \xi
\end{gathered}
$$

with

$$
\begin{aligned}
& K_{1}=6 R_{1}\left(\begin{array}{ccc}
1 & 1 & -4 \\
1 & 3 & -8 \\
-4 & -8 & 24
\end{array}\right), \quad K_{2}=6 R_{2}\left(\begin{array}{ccc}
1 & 1 & -4 \\
1 & 3 & -8 \\
-4 & -8 & 24
\end{array}\right) \text {. } \\
& V_{5}=G_{1}^{\mathrm{T}} Z G_{1}+G_{1}^{\mathrm{T}} Z G_{1}+G_{2}^{\mathrm{T}} Y G_{2}+G_{2}^{\mathrm{T}} Y G_{2} \\
& =\xi^{\mathrm{T}}\left[\begin{array}{llll}
2\left(e_{1}^{\mathrm{T}}\right. & e_{2}^{\mathrm{T}} & \tau^{2} e_{7}^{\mathrm{T}} & h^{2} e_{8}^{\mathrm{T}}
\end{array}\right) \\
& \times \mathrm{Z}\left(\begin{array}{lllllll}
e_{0}^{\mathrm{T}} & e_{4}^{\mathrm{T}} & \tau e_{1}^{\mathrm{T}}-\tau e_{5}^{\mathrm{T}} & h e_{1}^{\mathrm{T}}-h e_{6}^{\mathrm{T}}
\end{array}\right)^{\mathrm{T}}+2\left(\begin{array}{lllll}
e_{1}^{\mathrm{T}} & e_{2}^{\mathrm{T}} & \tau e_{5}^{\mathrm{T}} & h e_{6}^{\mathrm{T}}
\end{array}\right) \\
& \left.\times Y\left(\begin{array}{llll}
e_{0}^{\mathrm{T}} & e_{4}^{\mathrm{T}} & e_{1}^{\mathrm{T}}-e_{2}^{\mathrm{T}} & e_{1}^{\mathrm{T}}-e_{3}^{\mathrm{T}}
\end{array}\right)^{\mathrm{T}}\right] \xi
\end{aligned}
$$

From (16) to (21), one can see that

$$
V=\xi^{\mathrm{T}} \varphi \xi<0 .
$$

Obviously, the inequality (22) holds if the condition (8) from Theorem 3.1. Following the theory in [30], the system (1) is asymptotically stable.
Remark 3.1 In the proof of this theorem, the functional $V$ is general for considering much more information about the state vector. In particular, $V_{5}$ is very important to improve the delay-dependent stability condition. If one attempt to delete $x(t-\tau)$ from $G_{1}$ or $G_{2}$, the maximum of time-delay may less than those of original $V_{5}$. It is very important to use a new class of inequalities for improving delay-dependent stability condition. In short, the delay-dependent stability condition would be less conservative, which is shown in next section.

\section{EXAMPLE}

In this section, we will show the superiority of our approach in the above section according to the conventional systems.

Example 1. Consider the system (1) with the state matrices are listed as the following:

$$
A=\left(\begin{array}{cc}
-0.9 & 0.2 \\
0.1 & -0.9
\end{array}\right), \quad B=\left(\begin{array}{cc}
-1.1 & -0.2 \\
-0.1 & -1.1
\end{array}\right), \quad C=\left(\begin{array}{cc}
-0.2 & 0 \\
0.2 & -0.1
\end{array}\right),
$$

With different cases, we can obtain different maximum time delay $h$ as listed in Table 1 and Table 2, compared with some existing references.

The first case is $\tau \neq h$, the maximum of $h$ for different $\tau$ compared to the existing results are listed in Table 1 . Clearly, the maximum of $h$ using the Theorem 3.1 is very close to analytical bounds, which shows our approach is less conservative than the existing results. It is also shows that we could also obtain good condition without discrete Lyapunov functional approach.

The second case is $\tau=h$, the maximum of $h$ compared to the existing results are listed in Table 2 . Although our result is little less than that in [12], our condition is the best result except the condition obtain by using the approach in [12].

Furthermore, our approach may bring simple computation than that in [12]. Furthermore, using our approach may be more efficient to deal with neutral systems with mixed timevarying delay.

In a word, it is observed that our method presented in this paper is less conservative than most of the existing results.

TABLE I. THE MAXIMUM OF $h$ FOR DIFFERENT $\tau$

\begin{tabular}{l|c|c|c|}
\hline References & $\tau=0.1$ & $\tau=0.5$ & $\tau=1$ \\
\hline$[5]$ & 1.7100 & 1.6718 & 1.6543 \\
\hline$[6]$ & 1.7844 & 1.7495 & 1.7201 \\
\hline$[7]$ & 1.8307 & 1.7755 & 1.7213 \\
\hline$[12]$ & 2.1229 & 2.1229 & 2.1229 \\
\hline$[13]$ & 2.2951 & 2.3471 & 2.3752 \\
\hline $\begin{array}{l}\text { Theorem 3.1 } \\
\text { Analytical } \\
\text { bounds }\end{array}$ & 2.2959 & 2.3488 & 2.3769 \\
\hline
\end{tabular}


TABLE II. THE MAXIMUM OF $h$ AS $h=\tau$

\begin{tabular}{l|c|}
\hline References & $\tau=0.1$ \\
\hline$[21]$ & 1.3718 \\
\hline$[5]$ & 1.6527 \\
\hline$[14]$ & 1.7191 \\
\hline$[15]$ & 1.7220 \\
\hline$[6]$ & 1.7844 \\
\hline$[16]$ & 1.7856 \\
\hline$[7]$ & 1.8307 \\
\hline$[12]$ & 2.2254 \\
\hline Theorem 3.1 & 2.2250 \\
\hline Analytical bounds & 2.2255 \\
\hline
\end{tabular}

\section{CONCLUSION}

In this paper, an improved integral inequality has been introduced, as applications of this inequality, the delaydependent stability problems for neutral systems have been investigated. Based on a new constructed Lyapunov Krasovskii functional, and combined with the new integral inequalities, a new stability criterion has been obtained for neutral systems with mixed time-delay. Finally, one example has shown the effectiveness and less conservativeness of the condition presented in this paper.

\section{ACKNOWLEDGMENT}

This work was supported by the National Natural Science Foundation of China (Grant No.11461082), the National Natural Science Foundation of China(Grant No.61472093).

\section{REFERENCES}

[1] M.-W. Spong, “A theorem on neutral delay systems,” Systems \& Control Letters, vol. 6, issue. 4, pp. 291-294, October 1985.

[2] E. Fridman, U. Shaked, "Delay-dependent stability and $\mathrm{H} \infty$ control: constant and time-varying delays,” International Journal of Control, vol. 76, issue. 1, pp. 48-60, January 2003.

[3] M. Wu, Y. He, J.-H. She, "New delay-dependent stability criteria and stabilizing method for neutral systems," IEEE Transactions on Automatic Control, vol. 49, issue. 12, pp. 2266-2271, December 2004.

[4] Y. He, Q.-G. Wang, C. Lin, M. Wu, "Augmented Lyapunov functional and delay-dependent stability criteria for neutral systems," International Journal of Robust and Nonlinear Control, vol. 15, issue.18, pp. 923-933, December 2005

[5] Y. He, M. Wu, J.-H. She, G.-P. Liu, "Delay-dependent robust stability criteria for uncertain neutral systems with mixed delays,” Systems and Control Letters, vol. 51, no. 1, pp. 57-65, Jan 2004.

[6] X.G. Liu, M.Wu, R. Martin, M.L. Tang, "Stability analysis for neutral systems with mixed delays,” Journal Of Computational And Applied Mathematics. vol. 202, issue. 2, pp. 478-497, 15 May 2007.

[7] Wei Qian, Juan Liu, Youxian Sun, Shumin Fei, "A less conservative robust stability criteria for uncertain neutral systems with mixed delays," Mathematics and Computers in Simulation, vol. 80, issue.5, pp. 10071017, January 2010.

[8] Parlakci MNA, "Robust stability of uncertain neutral systems: a novel augmented Lyapunov functional approach,” IET Control Theory and Applications, vol. 1, issue. 3, pp. 802-809, May 2007.
[9] Q.-L. Han, "Robust stability of uncertain delay-differential systems of neutral type,” Automatica, vol. 38, issue. 4, pp. 719-723, April 2002.

[10] L.-L. Xiong, S.-M. Zhong, D.-Y. Li, "Novel delay-dependent asymptotical stability of neutral systems with nonlinear perturbations," Journal of Computational and Applied Mathematics, vol. 232, issue. 2, pp. 505-513, October 2009.

[11] Q.-L. Han, “A discrete delay decomposition approach to stability of linear retarded and neutral systems,” Automatica, vol. 45, issue.2, pp. 517-524, February 2009.

[12] Q.-L. Han, “On stability of linear neutral systems with mixed time delays A discretized Lyapunov functional approach,” Automatica, vol. 41, issue. 7, pp. 1209-1218, July 2005.

[13] Xu-Guang Li, Xin-Jian Zhu, Arben Cela, Abdellatif Reama, "Stability analysis of neutral systems with mixed delays,” Automatica, vol. 44, issue.11, pp. 2968-2972, November 2008.

[14] O.M. Kwon, J.H. Park, S.M. Lee, "On stability criteria for uncertain delay-differential systems of neutral type with time-varying delays," Applied Mathematics and Computation, vol. 197, issue. 2, pp. 864-873, 1 April 2008.

[15] S. Xu, J. Lam, Y. Zou, "Further results on delaydependent stability conditions of uncertain neutral systems," International Journal of Robust Nonlinear Control, vol. 15, issue. 5, pp. 233-246, 25 March 2005.

[16] Z. Zhao, W.Wang, B. Yang, "Delay and its time-derivative dependent robust stability of neutral control system,” Applied Mathematics and Computation, vol. 187,issue. 2, pp. 1326-1332, 15 April 2007.

[17] R. Krishnasamy, P. Balasubramaniam, “A descriptor system approach to the delay-dependent exponential stability analysis for switched neutral systems with nonlinear perturbations,” Nonlinear Analysis: Hybrid Systems, vol. 15, pp. 23-36, February 2015.

[18] S. Lakshmanan, T. Senthilkumar, P. Balasubramaniam, "Improved results on robust stability of neutral systems with mixed time-varying delays and nonlinear perturbations,” Applied Mathematical Modelling, vol. 35, issue. 11, pp. 5355-5368, November 2011.

[19] J. Sun, G.P. Liu, J. Chen, "Delay-dependent stability and stabilization of neutral time-delay systems," International Journal of Robust and Nonlinear Control, vol. 19, issue. 12, pp. 1364-1375, August 2009.

[20] M. Fang, Ju H. Park, “A multiple integral approach to stability of neutral time-delay systems,” Applied Mathematics and Computation, vol. 224, pp. 714-718, November 2013.

[21] Ju H. Park, O. Kwon, "On new stability criterion for delay-differential systems of neutral type,” Applied Mathematics and Computation, vol. 162, issue. 2, pp. 627-637, March 2005.

[22] Ju H. Park, “Novel robust stability criterion for a class of

neutral systems with mixed delays and nonlinear perturbations,” Applied Mathematics and Computation, vol. 161, issue 2, pp. 413-421, February 2005.

[23] M.-J. Park, O. Kwon, J.-H. Park, S.-M. Lee, E.-J. Cha, "Stability of timedelay systems viaWirtinger-based double integral inequality," Automatica, vol. 55, pp. 204-208, May 2015.

[24] A. Seuret, F. Gouaisbaut, "Wirtinger-based integral inequality: Application to time-delay systems," Automatica , vol. 49, issue. 9, pp. 2860-2866, September 2013.

[25] A. Seuret, F. Gouaisbaut, E. Fridman, "Stability of systems with fastvarying delay using improved Wirtinger's inequality,” 52nd IEEE conference on decision and control, Florence, Italy, pp. 946 - 951, 10-13 December 2013.

[26] A.Seuret, F.Gouaisbaut, "Complete quadratic Lyapunov functionals using Bessel-Legendre inequality," Proceedings of European Control Conference, Strasbourg, pp. 448-453, 24-27 June 2014.

[27] Y. He, Q. G. Wang, L. Xie, C. Lin. "Further improvement of freeweighting matrices technique for systems with timevarying delay," IEEE Transaction on Automatica Control, vol. 52, issue. 2, pp. 293-299, Feb 2007.

[28] P. Balasubramaniam, R. Krishnasamy, R. Rakkiyappan,

"Delay-dependent stability of neutral systems with time-varying delays using delay-decomposition approach,” Applied Mathematical Modelling, vol. 36, issue.5, pp. 2253-2261, May 2012. 
[29] P. G. Park,Won Il Lee, Seok Young Lee, “Auxiliary function based integral inequalities for quadratic functions and their applications to time-delay systems,” Journal of the Franklin Institute, vol. 352, issue. 4, pp. 1378-1396, April 2015.

[30] K. Gu, V.L.Kharitonov, J.Chen, "Stability of Time-Delay Systems," Birkh-user, Basel, 2003.

[31] R.-Q. Lu, H.-W. Wu, J.-J. Bai, “New delay-dependent robust stability criteria for uncertain neutral systems with mixed delays," Journal of the Franklin Institute, vol. 351, issue. 3, pp. 1386-1399, March 2014.

[32] S. Boyd, E.L. Ghaoui, E. Feron, V. Balakrishnan, "Linear matrix inequalities in systems and control theory,” Philadelphia: SIAM, 1994. 